\title{
Exploiting Program Execution Phases to Trade Power and Performance for Media Workload
}

\author{
Subhasis Banerjee, G. Surendra and S. K. Nandy \\ Supercomputer Education and Research Centre \\ Indian Institute of Science, Bangalore 560012 - INDIA \\ e-mail: \{subhasis@hamsadvani, surendra@rishi, nandy@\}.serc.iisc.ernet.in
}

\begin{abstract}
Processing streaming media comprises several program phases (often distinct) that are periodic and independent of application data. In this paper we characterize execution of such programs into execution phases based on their dynamic IPC (Instruction Per Cycle) profile. We show that program execution of selected phases can be dynamically boosted by activating additional standby functional units which are otherwise powered down for saving energy. Through simulation we show that speedup ranging from 1.1 to 1.25 can be achieved while reducing the energy-delay product (EDP) for most of the media benchmarks evaluated. Additionally we show that artificially introduced stalls during phases of processor underutilization reduces power by around 2 to $4 \%$.
\end{abstract}

\section{INTRODUCTION}

Runtime optimization of processor resources is becoming increasingly important from the perspective of performance and power. With the increasing complexity of the application programs in the domain of streaming media, the processing requirement is growing up [1]. High performance computing and embedded systems require high throughput with minimal power dissipation. During our study of program behavior analysis, we find that there exists distinct, periodic phases of execution characterized by Instruction Per Cycle (IPC), Reorder Buffer (RoB) occupancy, instruction issue rate, branch miss rate, cache miss rate etc. All these parameters show a definite pattern during execution when studied for a longer period of time. In this paper we show that the phased behavior of a program in terms of IPC and RoB occupancy, can be exploited to improve performance by allocating additional resources across different phases.

In our experiment with a four way issue superscalar processor we get IPC, averaged over entire execution of mpeg2decode program, just above 2, while the dynamic IPC averaged over 10,000 cycles varies from a minimum around 1.8 to a maximum around 2.7. This is shown in figure 1 . We observe that the program phases are control dominated as it remains invariant of the input data stream. In the figure each point in time axis represents average IPC over an interval of 10,000 cycles. The average variation of IPC over total execution time for the benchmarks studied is from $40 \%$ to $68 \%$ of the commit width. There is significant amount of ILP (Instruction Level Parallelism) not utilized by the processor due to resource constraint and/or true data dependence. In this paper we study the effect on execution time and the EDP by scaling the functional units and the instruction fetch window when the dynamic IPC attains a value over some threshold during program execution. Though the scaling of processor resources increases the power consumption, we find that the variation in EDP is negligible. We also study the effect on performance and power by introducing stalls when the processor resources are underutilized in different phases of program execution. The introduction of artificial stalls degrades the performance but it effectively manages the state of underutilization to save power and improve EDP.
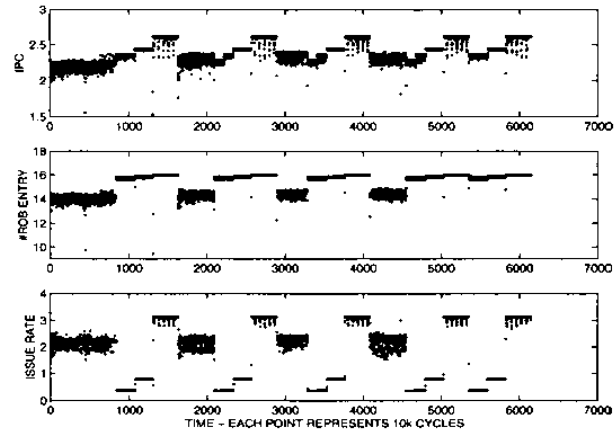

Fig. 1. Variation of dynamic IPC, number of RoB entry and number of instruction issued per cycle with time for mpeg2decode

We use SimpleScalar 3.0 [4] for the PISA instructions to simulate a dynamically scheduled superscalar processor. For power estimation we use Wattch [5], a performance and power analysis simulation tool that is built on top of SimpleScalar. It incorporates conditional clocking $(c c)$ at different levels. Table 1 shows the pipeline parameters used for carrying out simulations. The extended configuration parameters become effective only when we attempt to dynamically change the number of resources based on available parallelism. We use process parameters for a $.35 \mu \mathrm{m}$ process at $600 \mathrm{MHz}$ in all our simulations. We evaluate our ideas on a set of benchmarks from MiBench [6].

TABLE I

PROCESSOR PARAMETERS FOR base AND extended CONFGURATLONS IN OUR SIMULATIONS.

\begin{tabular}{|l||l|}
\hline Parameter & value \\
\hline \hline Fetch Queue/RUU/LSQ Size & $8 / 64 / 32$ instructions \\
\hline Fetch/Decode/Commit width & 4 instructions/cycle \\
\hline Issue width (OoO) & 4 instr/cycle $(8$ extended $)$ \\
\hline Functional Units & 4 int ALUs $(8$ extended $)$, \\
& 1 int mult/div $(2$ extended $)$ \\
& 2 mem ports $(4$ extended $)$ \\
\hline
\end{tabular}

II. Processor UNDERUTILIZATION IN TERMS OF PROGRAM PHASE

We observe that at any given cycle the number of instructions issued in issue stage of the pipeline is highly correlated with the number of instructions issued in the previous cycle and the number of instructions issued in the next cycle. Based upon this behavior, we compute average IPC in a window of execution cycles, which is a representative of the phase of the program bounded by that window. In our 


\section{$4 \mathrm{C}-4$}

experiment the number of cycles over which IPC is averaged to represent a state or phase of the processor, is 10,000 . When the processor enters into a state or phase it remains there for sometime and any measure taken to improve performance should sustain for that period of time. In the experiment we allocate resources for that period and update the phase information once in 10,000 cycle and decision for resource allocation is made based on updated state or phase information. From the behavior of variation of IPC, RoB occupancy, issue rate etc, we can infer that at some stages of execution the processor resources are underutilized. The smallest unit for which a resource configuration decision is made is taken as 10,000 cycles. A too small interval will lead to over-configuring the functional units and a too large interval will miss opportunities. While we implement throttling by stalling different pipeline stages, we consider the number of fetched, decoded or issued instructions in previous cycle as a parameter to make the decision. Resource scaling decision is taken based on the parameters representing the previous phase (averaged over 10,000 cycles).

\section{A. Fetch Decode and Issue Underutilization}

The fetch stage is said to be underutilized if the number of instructions that can be fetched per cycle from the instruction cache is less than the maximum fetch rate. The number of instructions that can be fetched per cycle is governed by the fetch width and number of free slots in the fetch queue. Stalls in subsequent pipeline stages results in slow clearance of the fetch queue leading to lower fetch rates. Our simulations indicate that the average fetch rate is 3.5 instructions/cycle (for the base configuration in table 1) for the benchmarks considered. The fetch stage fluctuates between normal and underutilized states depending on program behavior and available resources. In this paper we assume that the fetch stage is underutilized if 2 or fewer instructions can be fetched per cycle which corresponds to about $30 \%$ of all instruction fetches (not including zero instruction fetches).

The decode stage is underutilized if the decode bandwidth is not fully utilized due to non-availability of free RUU (Register Update Unit) slots. We assume decode stage is underutilized if 2 or fewer instructions are decoded. The issue stage is said to be underutilized if the number of instructions issued per cycle is less than the maximum issue rate. Issue underutilization occurs mainly due to true data dependencies and structural hazards.

\section{B. Functional Unit Underutilization}

During the phase of functional unit underutilization the instructions in pipeline wait for free functional unit. To improve performance (though at the cost of average power/cycle), we allocate or power on additional resources. These resources are in a standby mode (powered off or gated) during normal mode of processor operation. When the potential for increased parallelism exists, these resources are powered on (switched to an active mode) to cater to the additional demand thereby increasing the IPC. We use a scheme to power on/off the additional resources based on the following observations:-

(i) When the processor issues instructions at its maximum issue rate it continues to issue at the same (sometimes at nearly the same) rate for a certain number of cycles. It is observed that on average the processor issues 4 instructions/cycle more than $60 \%$ of the time. The additional resources are powered on (which are used in subsequent phases) if the IPC is above some threshold value in the previous phase. The resources are powered off if the maximum issue rate is not honored i.e. when the processor is underutilized.

(ii) Another heuristic that was evaluated was to turn on the additional resources when the number of instructions that are ready to be issued is greater than the issue width of the base configuration. In addition to increasing the resources, we impose fetch throttling (with fetch threshold set as $50 \%$ of fetch width, 2 cycles stall) which tends to reduce power marginally.

\section{Phase Based Optimization}

The method of our heuristics is to - $(i)$ allocate additional resources to the processor when it is in moderate or high IPC state, assuming that following phases of execution will extract more ILP, and (ii) throttle at different stage of pipeline when the processor is unable to fetch/decode/issue minimum number of instructions (in particular, 2 instruction) in the previous cycle.

\section{A. Power Management during Processor Underutilization}

Power dissipation increases with the increase in number of functional units and issue width. Power is proportional to the clock frequency of a processor. Power budget reduces when the frequency of operation reduces and effectively the performance degrades. So power is not an appropriate metric for comparing performance[2]. Energy is more appropriate than power and it is effectively the total area in a power-delay plot. Energy-Delay Product is the average energy consumed multiplied by the computation time required. It takes into account the trade off between delay and energy per operation. One can trade increased delay by supply voltage scaling for lower energy/operation. In this paper we choose EDP as a metric to evaluate the effectiveness of resource allocation across different program phases. The utility and impact of stalls on power in high-performance processors is affected by the accuracy of the branch predictor. Wrong path instructions that enter the pipeline consume additional power (up to $28 \%$ according to [3]) mainly in the rename logic, execution units (including caches), result bus and clock logic. To understand why introducing stalls at appropriate times improves power, we simulate the processor configuration in table 1 with the branch predictor replaced by a perfect predictor (see fig.2). This ensures that there are no branch mispredictions and no useless activities being performed by wrong path instructions.

\section{Fetch and Decode Throttling}

On a fetch stall, instruction cache, branch predictor and memory bus accesses are disabled (gated) resulting in lower power dissipation. It is seen that power dissipation is significantly smaller when the number of instructions in the fetch queue is 4 rather than 8 . This indicates that reducing the density of instructions in the fetch queue improves power. The throttling scheme does this by ensuring that the fetch queue is cleared to some extent before new instructions are fetched. Results indicate that a fetch threshold value of $70 \%$ of fetch width with a stall for $\mathrm{N}=2$ cycles results in about $1 \%$ decrease in speedup and $2 \%$ improvement in power. We introduce stalls if the number of instructions that can be decoded in the current cycle is less than a threshold. The decode stage is also stalled for the next $\mathrm{N}$ cycles if the number of instructions in the fetch queue is less than a specified threshold. We find that $\mathrm{N}=1$ gives a small improvement in the EDP. Simulations indicate that the contribution of RUU/LSQ sizes to decode stalls is almost negligible and most decode stalls are due to a limited decode width.

\section{Issue Throttling}

It is observed that one to three instructions are issued per cycle for $25 \%$ of the time. When no instructions are issued in any given cycle, the issue stage is gated and hence dissipates only the leakage power. In our simulations, we assume without loss of generality that the processor is underutilized when it issues one or two instructions per cycle which accounts for nearly $10 \%$ of all issues. Therefore, issue throttling is applied for about $10 \%$ of the time in the simulations. The general algorithm for the throttling scheme is :

if ( num_fetch+decode [ num_issue ] $>M)\{$ fetch+decode() [ issue() ]; \}

else \{ 
stall_fetch+decode_for_N_cycles(); [ stall_issue_for_N_cycles() ];

\}

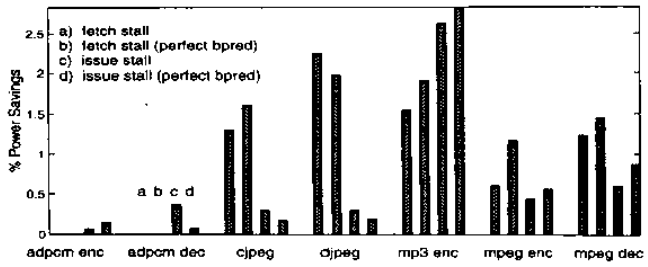

Fig. 2. Power savings (in \%) due to fetch and issue throttling with and without perfect branch prediction

Figure 2 shows the average power savings due to fetch and issue throttling with a stall of $N=2$ cycles. We set $M=2$ for our experiment. It is observed that the power savings with perfect branch prediction may be lesser than with imperfect prediction. For example, cjpeg with imperfect prediction (bar a) has lower power savings since $23 \%$ extra branch instructions are executed compared to just $10 \%$ in djpeg. Though perfect prediction improves IPC by avoiding useless activity (squashes), it tends to keep many of the queues (fetch queue, RUU etc) full most of the time resulting in larger power consumption in some benchmarks (e.g. djpeg). However, the EDP improves (is reduced) with perfect prediction in all benchmark programs. In contrast, the EDP with imperfect branch prediction does not improve in any of the benchmark programs indicating that pure issue throttling is not beneficial for saving energy.

\section{B. Resource Allocation with Throttling}

We study the runtime behavior of program in terms of IPC and issue rate during execution. As discussed in the previous section we identify the phase or state of the processor based upon dynamic IPC (averaged over 10,000 cycles). Additional resources are powered on when the processor attains a state where it records IPC more than $P$. Our study of behavior of different programs based on IPC, average RoB occupancy and average issue rate, shows that these parameters exhibit discontinuity in their profile (see figure 1). This behavior strengthen the scheme of selecting a threshold for transition from one state of processor configuration to another. The scheme for allocation of resources is as follows:

get_processor_state_id();

if

$(($ processor_state_id $==$ base $) \& \&$ dynamic_JPC $>P)$

switch_to_extended_state

else if

$(($ processor_state_id $==$ extended $) \& \&$ dynamic_IPC $<Q)$

switch_to_base_state;

When the processor is executing in the extended state the number of instructions in the ready queue should be higher to provide adequate number of instructions to the functional units. Non availability of the ready instructions due to true dependence at this phase will leave the functional unit idle and the power consumption will be higher and hence the processor is switched over to the base configuration. The choice of $P$ and $Q$ is made based on analysis of program phases for different benchmarks used in this experiment. When additional functional units are switched on, we expect ILP to be more than or equal to the issue width of base configuration. With this justification we select $Q$ as 4 . We set $P$ equal to 2.5 based on the fact that most of the benchmarks show ILP around 2. Figure 3 shows the normalized IPC, power and EDP with dynamic resource scaling (extended configuration) with and without fetch throttling. Bar (a) gives the base value with respect to which normalization is done. All benchmarks except adpcm show an average $1 \%$ to $3 \%$ power savings with negligible performance degradation. The EDP also decreases with throttling and resource allocation indicating that introducing stalls is beneficial for most benchmarks considered. With the extended configuration, the performance improves in all programs (very little improvement in adpcm due to limited parallelism) with increased power dissipation. JPEG decoding yields the best improvement in the EDP (15\% reduction) with a $25 \%$ improvement in speedup and $35 \%$ extra power dissipation for the extended configuration.

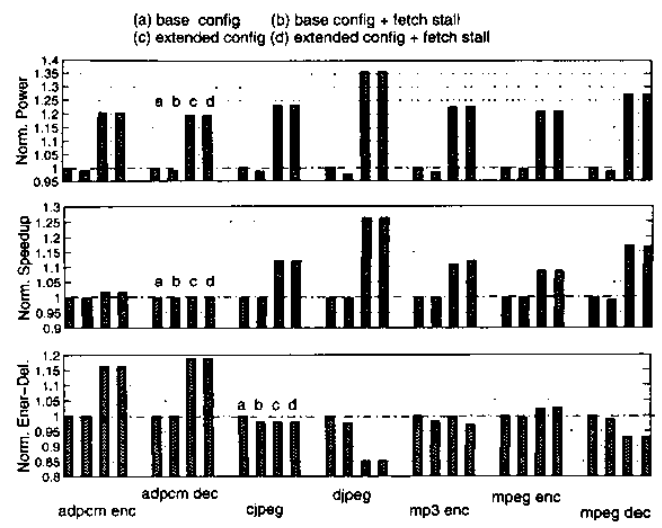

Fig. 3. Speed up and EDP with Resource Allocation in Presence of Throttling

\section{CONCLUSIONS AND FUTURE WORK}

In this paper we analyzed the phased behavior of program to determine the execution state of processor and quantify it in terms of IPC and issue rate. Optimizations by incorporating resource scaling with stall at pipeline stages, produced improvement in EDP with speedup by 1.15 on average. Runtime optimization of execution speed and processing power depends on efficient detection of program phases. Power, which is an important design parameter, depends on the instruction density in pipeline stages. Effective utilization of resources minimize the occupancy of instruction in the pipeline. In our scheme additional stalls clear the pipeline congestion and on average $10 \%$ power can be saved with almost equal $(10 \%)$ degradation in performance. Through this work we establish the need for automatic identification of program phases and corresponding micro-architectural support to optimize these program phases at runtime.

\section{REFERENCES}

[1] K. Diefendorff, P. K. Dubey, "How multimedia workloads will change processor design," In IEEE computer, Vol 30 Issue 9, Sept. 1997

[2] R. Gonzalez, and M. Horowitz, "Energy Dissipation in General Purpose Microprocessors," In IEEE Journal of Solid State Circuits, 31(9), pp. 1277-1284, 1996.

[3] D. Folegnani and A. Gonzalez, "Energy-Effective Issue Logic," In Proc. of the Int. Symp. on Computer Architecture, 2001.

[4] D. Burger, T.M Austin, and S. Bennet, "Evaluating Future Microprocessors: The SimpleScalar Tool Set," Technical Report CS-TR-96-1308, University of Wisconsin-Madison, July 1996.

[5] D. Brooks, V. Tiwari, and M. Martonosi, "Wattch: A framework for Architectural-Level Power Analysis and Optimization," In Proc. of $27^{\text {th }}$ Intn'l. Symp. on Computer Architecture, June 2000.

[6] M. Guthaus, J. Ringenberg, D. Ernst, T. Austin, T. Mudge, R. Brown, "MiBench: A free, commercially representative embedded benchmark suite," In IEEE 4th Annual Workshop on Workload Characterization, Dec 2001. 